\title{
Human Platelets Express Functional Thymic Stromal Lymphopoietin Receptors: a Potential Role in Platelet Activation in Acute Coronary Syndrome
}

\author{
Boyuan Wang ${ }^{a, c}$ Yudong Peng ${ }^{a, c}$ Jiangchuan Dong ${ }^{a, c}$ Jing Lin ${ }^{a}$ Chun Wu ${ }^{a}$ \\ Yousu Su ${ }^{b}$ Hongcheng Fang ${ }^{b}$ Lei Wang $^{\text {b Kai Huang }}{ }^{\mathrm{a}}$ Dazhu Li ${ }^{\mathrm{a}}$ \\ aDepartment of Cardiology, Institute of Cardiovascular Diseases, Union Hospital, Tongji Medical \\ College, Huazhong University of Science and Technology, Wuhan, 'Department of Cardiology, \\ Shenzhen Sixth People's Hospital (Nanshan Hospital), Huazhong University of Science and Technology, \\ Union Shenzhen Hospital, Shenzhen, China; 'Boyuan Wang, YudongPeng and Jiangchuan Dong \\ contributed equally to this work
}

\section{Key Words}

Thymic stromal lymphopoietin • Inflammation • Platelets • Acute coronary syndrome.

\begin{abstract}
Background: Thymic stromal lymphopoietin (TSLP) has been shown to be expressed in various inflammatory tissues, such as human atherosclerotic plaques. Many types of myeloid cells involved in atherosclerosis, including mast cells, lymphocytes, dendritic cells and monocytes/macrophages, present TSLP receptors (TSLPR). However, it is unknown whether platelets, which also play important roles in atherothrombosis, express TSLPR. Methods and Results: We applied flow cytometry and western blotting to show that TSLPR was expressed on the surface of human platelets. Following the addition of TSLP to platelets, the expression of CD62P, CD63, PAC-1 and p-Akt as well as aggregation and ATP release were increased significantly. A TSLPR antibody and a PI3K (phosphatidylinositol 3-kinase) enzyme inhibitor (LY294002) significantly inhibited the platelet activation induced by TSLP. The expression of TSLPR, CD62P and CD63 and the increment of the expression of CD62P and CD63 induced by TSLP in the acute coronary syndrome (ACS) group were markedly higher than those in the control group and the stable angina pectoris (SAP) group. The expression and the increment of the expression of CD62P and CD63 induced by TSLP were positively correlated with the expression of TSLPR. Conclusion: Human platelets express functional TSLPR, which can be activated by TSLP to promote platelet activation. TSLP/TSLPR functions via activating the PI3K/AKT pathway, and this signalling pathway may be one of the mechanisms involved in thrombosis in ACS. In coronary disease patients, the determination of TSLPR in platelets may help to identify the risk of ACS.
\end{abstract}

Copyright $(2013$ S. Karger AG, Basel 


\section{Introduction}

Platelet activation and thrombosis play important roles in the pathogenesis of acute coronary syndrome (ACS) and stroke [1-5]. There are many mechanisms and cellular factors involved in platelet activation and thrombosis. Inflammation and inflammatory mediators are vital for thrombosis and atherosclerotic coronary artery disease [3, 6, 7]. Thymic stromal lymphopoietin (TSLP) mediates inflammation and is essential for allergic inflammation. In a preliminary study, we showed that TSLP plays an important role in the innate and adaptive immune system and is over-expressed in human atherosclerotic plaques $[8,9]$.

Thymic stromal lymphopoietin (TSLP) is an IL-7-like cytokine that was cloned from a murine thymic stroma cell line [10]. The gene encoding TSLP in humans is located at chromosome $5 q 22.1$, next to the atopic cytokine cluster at 5q31. In recent years, many studies have made significant progress in research on the cellular sources and stimuli inducing TSLP expression $[11,12]$. The TSLP receptor (TLSPR) is an approximately $50 \mathrm{kDa}$ protein that shows significant similarity to the common $\gamma$-chain $[13,14]$. TSLP signalling occurs via TSLPR expressed on various cell types of the innate and adaptive immune system. It mediates various types of allergic inflammation, such as atopic dermatitis and asthma, and plays an important role in the polarisation of dendritic cells (DCs) to drive T helper (Th) 2 cytokine production [11, 12, 15-17]. Ox-LDL can induce TSLP expression in human vascular smooth muscle cells (HVSMCs) and human umbilical vein endothelial cells (HUVECs), and angiotensin II can induce primary vascular smooth muscle cells (VSMCs) to express TSLP $[8,9]$. In addition, TSLP can promote the Th17 immune response in vitro, which may be implicated in Th17 inflammation in atherosclerosis through DC activation; Angiotensin II-induced TSLP enhances the Th17-driven immune response in atherosclerosis and hypertension $[8,9]$.

Many types of myeloid and lymphoid cells express TSLPR, such as monocytes, dendritic cells, T-cells, and B-cells $[13,14,18-20]$, which are also involved in atherosclerosis. In addition, TSLP exerts its functions via the PI3K (phosphatidylinositol 3-kinase)/AKT pathway [21, 22], which is also implicated in platelet activation [23-25]. Therefore, we designed this study to explore whether platelets, which originate from myeloid cells as megakaryocytes and play roles in atherothrombosis, express TSLPR and the potential role of the TSLP/TSLPR signalling channel in platelet activation.

\section{Materials and Methods}

\section{Study population}

The study subjects were divided into three groups: a stable angina pectoris (SAP) group, acute coronary syndrome (ACS) group and healthy controls. The SAP and ACS subjects were chosen from patients with suspected coronary artery disease (CAD), who therefore received coronary angiography. The healthy control group consisted of apparently healthy people who visited hospital for physical examination. The SAP group included 23 people; the ACS group, 22 people; and the healthy control group, 20 people. All subjects were recruited from Union Hospital of Tongji Medical College, Huazhong University of Science and Technology (HUST), Wuhan, China. The exclusion criteria were as follows: an age below 18 years, a lack of informed consent, and presenting with advanced liver disease, chronic-immune mediated diseases, renal failure, thromboembolism or infections during last four weeks. The age and sex were matched between all groups. The demographic details are given in Table 1.

\section{Ethics}

This research was approved by the Ethics Committee of Union Hospital of Tongji Medical College, Huazhong University of Science and Technology. All patients provided written informed consent and the study, abided by the guidelines of the Helsinki declaration and its amendments. 
Table 1. Clinical Characteristics. Data are expressed as numbers and percentages. ACS = acute coronary syndrome; $\mathrm{SAP}=$ stable angina pectoris, $\mathrm{CTRL}=$ control subjects. $\mathrm{CAD}=$ coronary artery disease; $\mathrm{ACE}=$ angiotensin-converting enzymes

\begin{tabular}{|c|c|c|c|}
\hline Characteristics & ACS $(n=22)$ & SAP $(n=23)$ & CTRL $(n=20)$ \\
\hline Aga, years $( \pm$ SD $)$ & $65.3 \pm 6.7$ & $63.8 \pm 10.3$ & $66.7 \pm 12.1$ \\
\hline Female, n (\%) & $5(22.7)$ & $6(26.1)$ & $5(25)$ \\
\hline \multicolumn{4}{|l|}{ Risk factors } \\
\hline No risk factors & $2(9.1)$ & $3(13.0)$ & $7(35)$ \\
\hline Hypertension, n (\%) & $18(81.8)$ & $18(78.3)$ & $5(25)$ \\
\hline Diabetes mellitus, $\mathrm{n}$ (\%) & $8(36.4)$ & $7(30.4)$ & 1 (5) \\
\hline Smoking, $\mathrm{n}(\%)$ & $8(36.4)$ & $9(39.1)$ & $5(25)$ \\
\hline Obesity, n (\%) & $6(27.3)$ & $8(34.8)$ & $3(15)$ \\
\hline Hyperlipidaemia, n (\%) & $13(59.1)$ & $16(69.6)$ & $4(20)$ \\
\hline \multicolumn{4}{|l|}{ CAD, no. (\%) } \\
\hline One vessel & $6(27.3)$ & $3(13.0)$ & - \\
\hline Two vessels & $7(31.8)$ & $11(47.8)$ & - \\
\hline Three vessels & $9(40.9)$ & $9(39.1)$ & - \\
\hline \multicolumn{4}{|l|}{ Medication, no.(\%) } \\
\hline No medication & $4(18.2)$ & $10(43.5)$ & - \\
\hline ACE-inhibitor & $12(54.5)$ & $8(34.8)$ & - \\
\hline AT-receptor blockers & $3(13.6)$ & $3(13.0)$ & - \\
\hline$\beta$-Blockers & $14(63.6)$ & $11(47.8)$ & - \\
\hline Calcium blocker & $7(31.8)$ & $5(21.7)$ & - \\
\hline Statins & $14(63.6)$ & $9(39.1)$ & - \\
\hline Aspirin & $15(68.2)$ & $10(43.5)$ & - \\
\hline Clopidogrel & $9(40.9)$ & $7(30.4)$ & - \\
\hline Vit $\mathrm{K}$ antagonist & $2(9.1)$ & $2(8.7)$ & - \\
\hline
\end{tabular}

\section{Definitions}

The AHA/ACC guidelines were used to diagnose coronary heart disease.

SAP: stable angina pectoris symptoms, without progression and stable intensity.

ACS: Episodes of $>20$ min of angina pectoris, with progress over time, or with recurrent episodes at rest, or with minimal physical strain within the last $24 \mathrm{~h}$. The electrocardiogram (ECG) shows at least one of the following criteria: a new ST-depression of at least $0.1 \mathrm{mV}$ or a negative $\mathrm{T}$ of at least $0.3 \mathrm{mV}$ in two or more leads of a standard ECG. Creatine kinase (CK) and Troponin-I levels may be increased, but can be normal as well.

STEMI: Episodes of angina pectoris of $>20 \mathrm{~min}$, with the following ECG and laboratory criteria: STsegment elevation in two or more leads with at least a $0.1 \mathrm{mV}$ or two-fold elevation of CK compared with normal levels and with a significant CK-MB and/or increased Troponin-I level.

NSTEMI: Episodes of angina pectoris of $>20 \mathrm{~min}$, with the following ECG and laboratory criteria: no STsegment elevation in a standard ECG, two-fold elevation of CK compared with normal levels and a significant CK-MB or increased Troponin-I.

\section{Preparation of human platelets}

Blood samples were drawn into plastic tubes containing 3.8\% sodium citrate and centrifuged at 180 g for 10 min to obtain platelet-rich plasma (PRP). Washed platelet (WP) suspensions were obtained via centrifugation (1,000 g, $10 \mathrm{~min}$ ) of PRP in the presence of prostacyclin [PGI (prostaglandin $\left.\left.\mathrm{I}_{2}\right)\right](75 \mathrm{nM}$ ), and the platelets were then washed in Tyrode buffer $\left(145 \mathrm{mM} \mathrm{NaCl}, 5 \mathrm{mM} \mathrm{KCl}, 0.5 \mathrm{mM} \mathrm{Na} \mathrm{HPO}_{4}, 1 \mathrm{mM} \mathrm{MgSO}\right.$, 10 mM HEPES, 5 mM glucose, PH 6.5). The WPs were subsequently resuspended in Tyrode buffer, and the number of platelets was adjusted to $3 \times 10^{8}$ cells $/ \mathrm{ml}^{-\mathrm{Ca}^{2+}}(1 \mathrm{mM})$ was added prior to stimulation experiments. Highly purified platelets, which were employed for the detection of TSLPR expression, were obtained via depletion of leucocytes using a high-efficiency leuco reduction filter (Purecell PL, PallBiomedical Products, USA) [26].

\section{Flow Cytometry}

Highly purified platelets were stained with a PE-conjugated anti-TSLP antibody (eBioscience, USA) and fixed in $0.5 \%$ paraformaldehyde. The expression of cell-surface TSLPR in human platelets was detected via flow cytometry (FCM). To evaluate the effect of the TSLP/TSLPR signalling channel on platelet activation, 
WPs obtained from healthy people were incubated separately with an anti-TSLPR antibody $(20 \mu \mathrm{g} / \mathrm{ml})$ (R\&D Systems, USA), a PI3K inhibitor (LY294002, purchased from Cayman Chemical, USA) or an equal amount of vehicle for 20 mins, followed by treatment with TSLP (5 $\mathrm{g} / \mathrm{ml}$ ) (Peprotech, USA) for $20 \mathrm{~min}$. Platelets in the control group were treated with vehicle alone. After staining with a PE-conjugated antiCD62P antibody (Biolegend, USA), PE-conjugated anti-CD63 antibody (Biolegend, USA) or FITC-conjugated PAC-1 (BD Biosciences, USA), which binds to and fixes activated GPIIb/IIIa integrin, the WPs were analysed by FCM. To analyse the effect of TSLP on platelet activation in CAD patients and healthy controls, the mean fluorescence intensity (MFI) of CD62P and CD63 was detected, and the increase in MFI compared with the platelets that were not stimulated by TSLP was recorded. Relative isotype controls were employed to enable correct compensation and confirm antibody specificity. Each measurement was performed in duplicate.

\section{Platelet aggregation and ATP release}

Platelet aggregation and ATP release were measured in a lumi-aggregometer (Chrono-Log Corporation, USA). $\mathrm{Ca}^{2+}(1 \mathrm{mM})$ was added to the washed platelets, which were then incubated separately with TSLP $(50 \mu \mathrm{g} / \mathrm{ml})$, collagen $(16 \mu \mathrm{g} / \mathrm{ml})$ (Chrono-Log Corporation, USA), TSLP $(50 \mu \mathrm{g} / \mathrm{ml})+$ collagen $(16 \mu \mathrm{g} / \mathrm{ml})$, or an equal amount of vehicle as a control. Platelet aggregability was evaluated according to the maximal percentage of platelet aggregation [26]. ATP levels were measured at the end of the assay by adding a known amount of an ATP standard $(2 \mu \mathrm{M})$ [26]. Five independent experiments were performed with different donor platelets. Each measurement was performed in duplicate.

\section{Western Blotting}

Following the aggregation reaction, the platelets were centrifuged. The precipitated platelets were washed twice with PBS and then lysed in lysis buffer. The lysates were centrifuged and denatured for 10 minutes. The protein samples were subsequently separated via SDS-PAGE and analysed by Western blotting. The primary antibodies used in these analyses included an AKT (phosphor-ser473) antibody (1:500, Santa Cruz Biotechnology, USA) and a $\beta$-actin antibody (1:1000, Sigma, USA). Nitrocellulose membranes were employed for protein transfer, and a chemiluminescence reagent (ThermoScientific, USA) was used to detect the chemiluminescent signal. Western blotting was also used for the detection of TSLPR expression in highly purified platelets. These platelets were run on gels and blotted with a human-TSLPR antibody or $\beta$-actin antibody. Peripheral blood mononuclear cells (PBMC) served as a positive control.

\section{Statistical analysis}

The results are presented as the mean \pm SEM. Statistical analyses of the experimental results from the SAP, ACS and CTRL groups were performed using an unpaired Student's t test. Student's t paired test was employed to determine the significance of differences observed in the TSLP stimulation experiment. The correlation between two variables was tested via Spearman correlation. $\mathrm{P}<0.05$ was considered statistically significant. All statistical analyses were performed with SPSS 13.0 (SPSS Inc, Chicago, IL).

\section{Results}

Expression of cell-surface TSLPR in human platelets

We used flow cytometry to detect the expression of cell-surface TSLPR in human platelets. The rate of TSLPR positivity in healthy people $(n=5)$ was $9.41 \pm 0.53 \%$ (Fig. 1). TSLPR expression in platelets was analysed by Western blotting. Highly purified platelets were blotted with a human-TSLPR antibody (R\&D) or a $\beta$-actin antibody. Peripheral blood mononuclear cells (PBMC) served as a positive control. This result showed that TSLPR was expressed in human platelets (Fig. 1).

TSLPR can be activated by TSLP to induce platelet activation and inhibited by a PI3K inhibitor

To test whether TSLPR functions in platelet activation, an anti-TSLPR antibody (R\&D Systems, USA) and TSLP were incubated with washed platelets. A PI3K inhibitor (LY294002) was used to explore the role of the PI3K/AKT pathway in this reaction. The mean fluorescence 


\section{Cellular Physiology $\quad$ Cell Physiol Biochem 2013;32:1741-1750 and Biochemistry \\ Wang et al.: A Potential Role of Thymic Stromal Lymphopoietin in Platelet Activation in Acute Coronary Syndrome}

Fig. 1. Expression of TSLPR in human platelets. (A) The expression of cell-surface TSLPR in human platelets was analysed via flow cytometry. The rate of TSLPR positivity in healthy individuals $(\mathrm{n}=5)$ was $9.41 \pm 0.53 \%$. Representative data are shown. The results are presented as the mean \pm SEM. (B) TSLPR expression in platelets was analysed by Western blotting. Peripheral blood mononuclear cells (PBMCs) served as a positive control. These results showed that TSLPR is expressed in platelets.

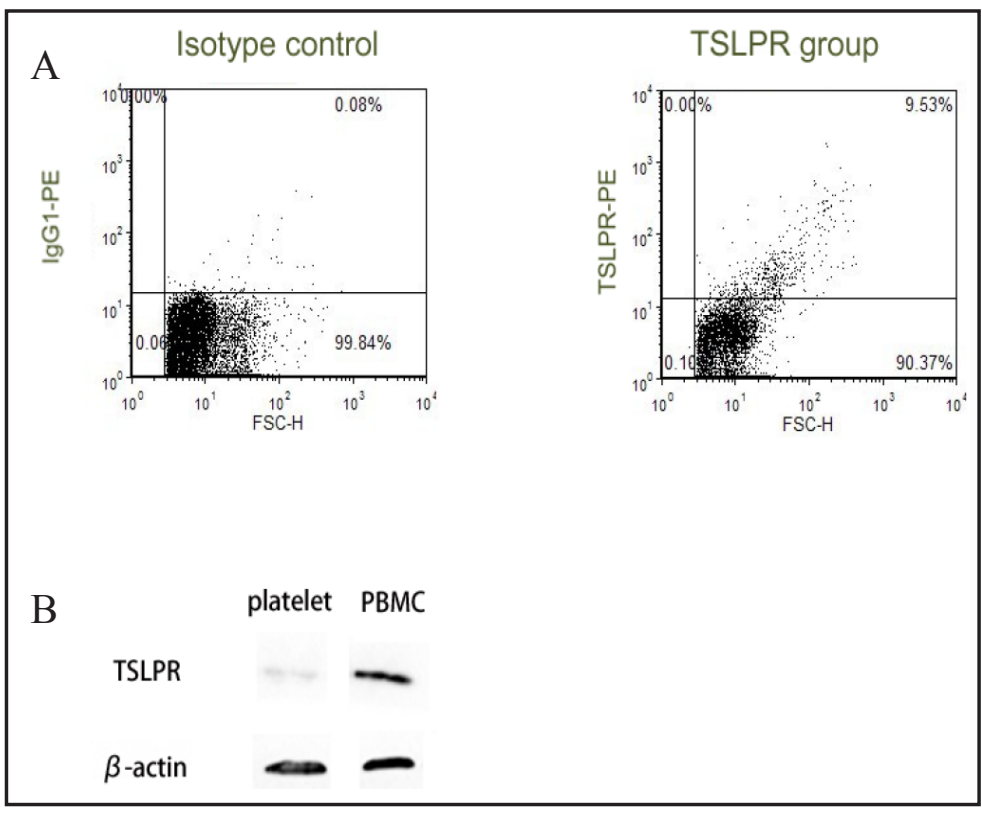

Fig. 2. TSLPR can be activated by TSLP to induce platelet activation and inhibited by a PI3K inhibitor. The results of statistical analysis of the mean fluorescence intensity (MFI) of CD62P, CD63 and PAC-1 in the CTRL, TSLP, TSLP+anti-TSLPR and TSLP+LY294002 groups are shown. Compared with the other groups, the expression of CD62P, CD63 and PAC-1 in

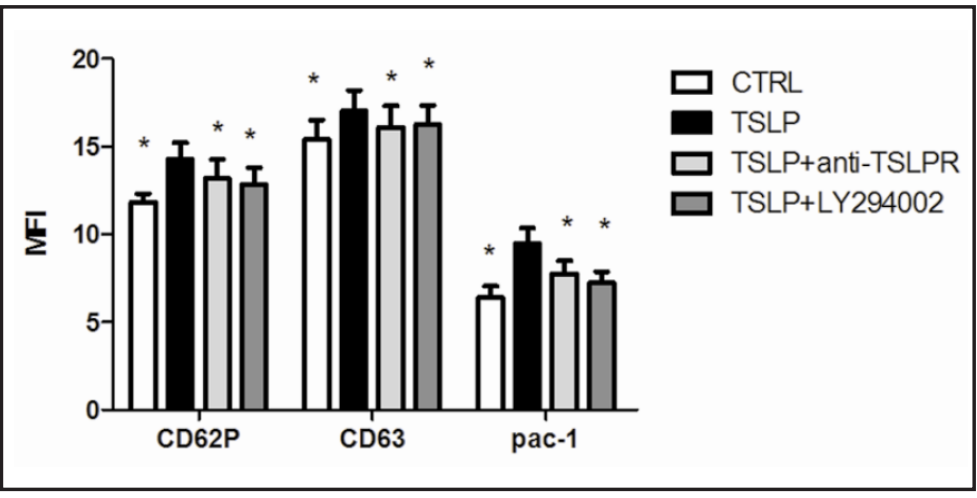

the TSLP-stimulated group was significantly enhanced. This result shows that TSLP can induce platelet activation, and an anti-TSLP antibody or PI3K inhibitor can inhibit the platelet activation induced by TSLP. *means, vs. the TSLP group, $\mathrm{p}<0.05$; the results are shown as the mean \pm SEM. The results are from five independent experiments with different donor platelets.

intensity (MFI) of CD62P, CD63 and PAC-1 was detected. Five independent experiments were performed using different healthy donor platelets. Each measurement was performed in duplicate.

The MFIs of CD62P, CD63 and PAC-1 in platelets stimulated by TSLP alone were significantly higher than in platelets without any intervention, or those that were preincubated with anti-TSLPR or LY294002 (Fig. 2). These results showed that TSLP could induce platelet activation and the anti-TSLP antibody and PI3K inhibitor inhibited the platelet activation induced by TSLP.

\section{Effect of TSLP on platelet aggregation and ATP release}

A lumi-aggregometer was used to test the effect of TSLP on platelet aggregation and ATP release. In the first experiment, we found that TSLP $(50 \mu \mathrm{g} / \mathrm{ml})$ was able to induce platelet aggregation and ATP release. We added collagen to the washed platelets to induce them to release sufficient amounts of adhesive macromolecules, such as fibrinogen, which is required for platelet aggregation, and found that TSLP significantly enhanced 
Fig. 3. The effect of TSLP on platelet aggregation and ATP release. TSLP alone can induce platelet aggregation and ATP release, $\mathrm{P}<0.05$ vs. CTRL. Compared with the collagenalone group, the maximum platelet aggregation rate in the TSLP+collagen group was significantly increased $(\mathrm{p}<0.05)$, and the level of ATP was also significantly increased $(p<0.05)$. The results are from five independent experiments with different donor platelets. Representative data are shown.

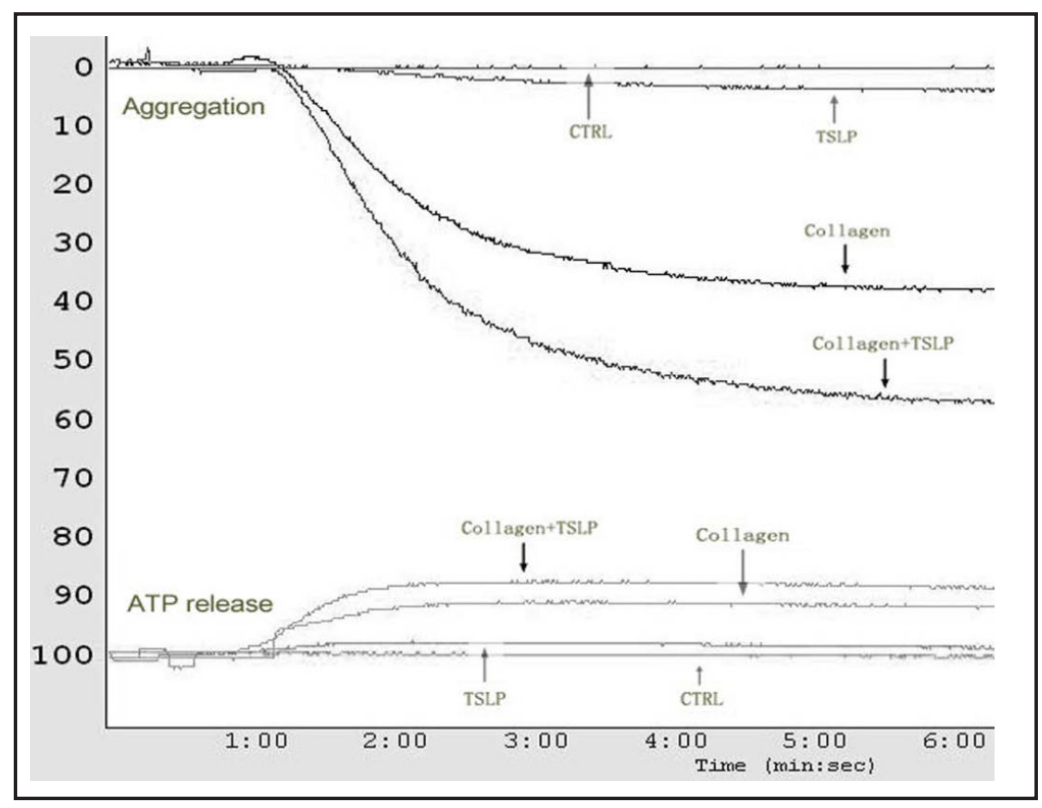

Fig. 4. The effect of TSLP on AKT phosphorylation in platelets. TSLP is able to markedly enhance AKT phosphorylation. The level of platelet p-AKT in the collagen group was higher than in the TSLP group. The level of AKT phosphorylation was significantly increased in platelets stimulated with TSLP and collagen compared with those stimulated with collagen alone. The results are from five independent experiments with different donor platelets. Representative data are shown.

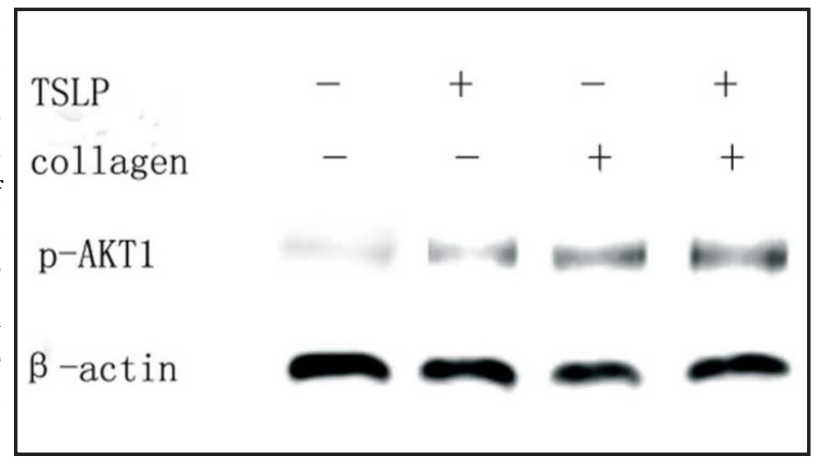

collagen-induced platelet aggregation. Then, five independent experiments using different donor platelets from healthy controls were performed to confirm this finding, and each measurement was performed in duplicate. Platelet aggregability was evaluated based on the maximal percentage of platelet aggregation.

The maximal percentage of platelet aggregation was $4.3 \pm 1.1 \%$ in platelets stimulated with TSLP alone; $39.2 \pm 4.3 \%$ in platelets stimulated with collagen alone; $58 \pm 6.8 \%$ in platelets stimulated with TSLP together with collagen; and $0.0 \pm 0.0 \%$ in the control group. The concentration of ATP was $0.21 \pm 0.05 \mu \mathrm{M}$ in platelets stimulated with TSLP alone; $2.09 \pm 0.28$ $\mu \mathrm{M}$ in platelets stimulated with collagen alone; $2.60 \pm 0.23 \mu \mathrm{M}$ in platelets stimulated with TSLP together with collagen; and $0.0 \pm 0.0 \%$ in the control group. The results are shown as the means \pm SEM. $p<0.05$ between each pair of groups (Fig. 3 ).

Effect of TSLP on the levels of phosphorylated AKT ( $p-A K T$ ) in platelets

Following the aggregation reaction, the platelets were analysed by Western blotting. We found that TSLP was able to enhance AKT phosphorylation markedly compared to the control. The level of AKT phosphorylation was significantly increased in platelets stimulated with TSLP together with collagen compared with that in platelets stimulated with collagen alone (Fig. 4).

\section{Expression of TSLPR in platelets of CAD patients}

We detected the expression of TSLPR in CAD patients and healthy individuals via flow cytometry. The rate of TSLPR positivity in platelets from the healthy control group $(n=20)$ 


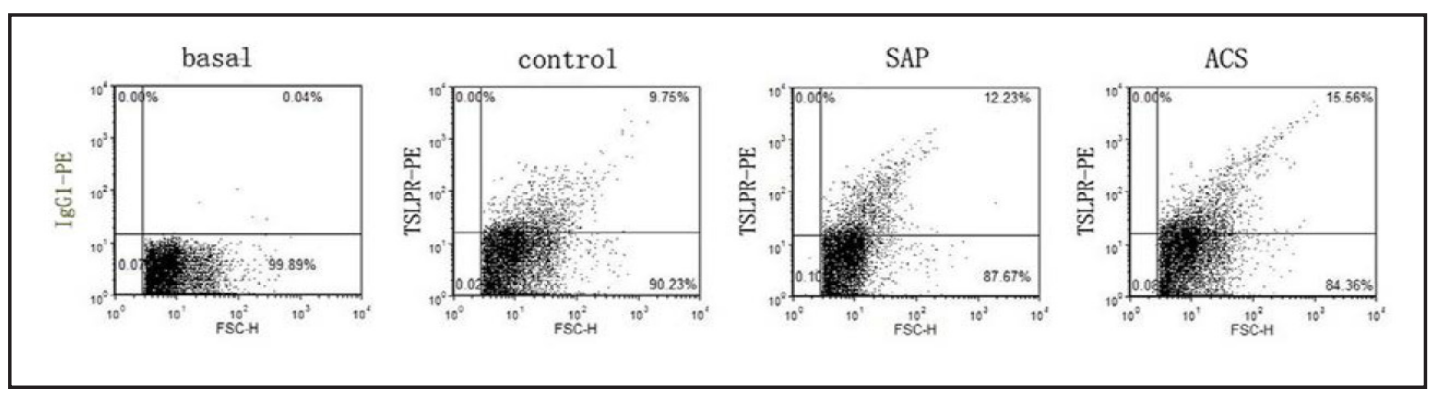

Fig. 5. The expression of cell-surface TSLPR in platelets from CAD patients was analysed via flow cytometry. The rate of platelet TSLPR positivity in the control group was $9.43 \pm 0.31 \%$; the positive rate in the SAP group was $12.04 \pm 0.52 \%$; and the positive rate in the ACS group was $15.98 \pm 0.67 \%$. SAP group vs. control group, $\mathrm{p}<0.05$; ACS group vs. control group, $\mathrm{p}<0.05$; SAP group vs. ACS group, $\mathrm{p}<0.05$; Representative data are shown. The results are shown as the mean \pm SEM.

Fig. 6. Expression of TSLPR correlated with platelet activation. (A) The MFI of CD62P in the different groups; (B) the MFI of CD63 in the different groups; (C) correlation analysis of TSLPR and CD62P expression $(r=0.19, P<0.01)$; (D) correlation analysis of TSLPR and CD62P expression, ( $\mathrm{r}=0.15$, $\mathrm{P}<0.01)$. * means vs. the control group, $\mathrm{p}<0.05 ; \Delta$ means vs. the SAP group, $\mathrm{p}<0.05$. The results are shown as the mean \pm SEM.
A

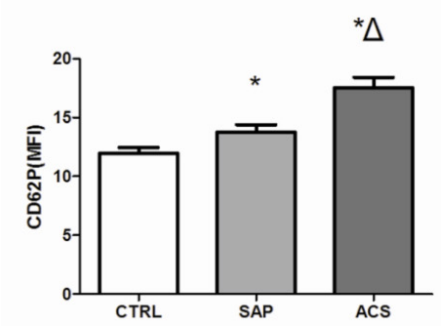

C

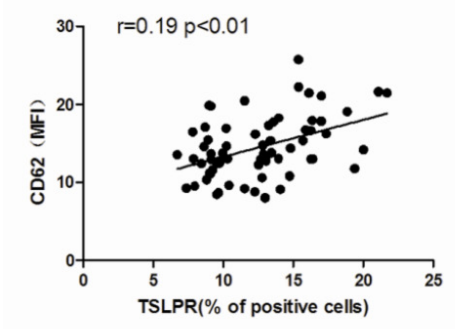

B

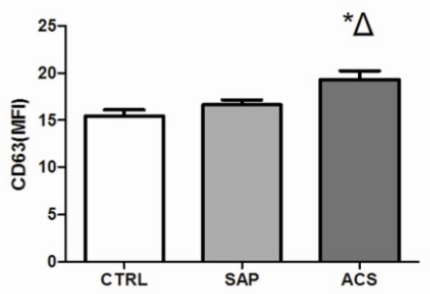

D

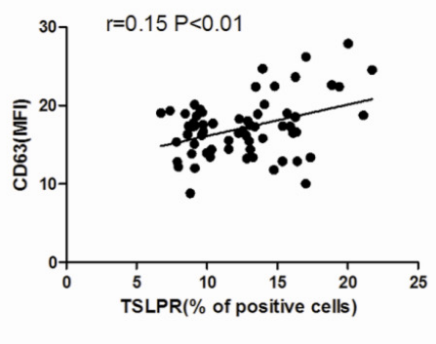

was $9.43 \pm 0.31 \%$, while the positive rates in the SAP group $(n=23)$ and ACS group $(n=22)$ were $12.04 \pm 0.52 \%$ and $15.98 \pm 0.67 \%$, respectively.

\section{Expression of TSLP correlated with platelet activation}

We consecutively analysed the expression of CD62P and CD63 in platelets from the CAD group and healthy controls via flow cytometry. Each measurement was performed in duplicate, and the mean fluorescence intensity was used as an index of CD62P and CD63 expression. We found that the expression of CD62 and CD63 in the platelets of the ACS group $(\mathrm{n}=22)$ was markedly higher than in the control group $(\mathrm{n}=20)$ and the SAP group $(\mathrm{n}=23)$. MFI of CD62P in ACS vs. CTRL: $17.55 \pm 0.87$ vs. $11.97 \pm 0.50$, $p<0.05$; and MFI of CD63: $19.27 \pm 0.97$ vs. $15.43 \pm 0.64, p<0.05$. Similarly, MFI of CD62P in ACS vs. SAP: $17.55 \pm 0.87$ vs. $13.78 \pm 0.63$, $\mathrm{p}<0.05$; and MFI of CD63: $9.27 \pm 0.97$ vs. $16.62 \pm 0.55$, $\mathrm{p}<0.05$. The expression of CD62P in the SAP group was markedly higher than that in the controls. MFI of CD62P in SAP vs. CTRL was $13.78 \pm 0.63$ vs. $11.97 \pm 0.50, p<0.05$. The expression of CD63 did not differ between the 
controls and the SAP group. MFI of CD63 in SAP vs. CTRL was $16.62 \pm 0.55$ vs. $15.43 \pm 0.64, p=$ 0.16 . The results are presented as the means \pm SEM. In addition, the expression of CD62P and CD63 was positively correlated with the expression of TSLPR. Correlation analysis of the expression TSLPR and CD62P: $r=0.19, \mathrm{p}<0.01$; correlation analysis of the expression of TSLPR and CD63 expression: $r=0.15, \mathrm{p}<0.01$ (Fig. 6, A-D).

\section{Effect of TSLP on platelet activation in CAD patients}

The subsequent experiment in the CAD group and healthy controls was carried out. Following stimulation by TSLP, the expression of CD62P and CD63 was detected via flow cytometry. An increase in the MFI of CD62P and CD63 was observed in platelets stimulated by TSLP compared with unstimulated platelets. Each measurement was performed in duplicate.

The increase in CD62 and CD63 expression in the ACS group ( $\mathrm{n}=22)$ was markedly higher than in the controls $(\mathrm{n}=20)$ and the SAP group $(\mathrm{n}=23) . \triangle \mathrm{CD} 62 \mathrm{P}$ in ACS vs. CTRL was $3.91 \pm 0.24$ vs. $2.29 \pm 0.18, \mathrm{p}<0.05$; and $\Delta \mathrm{CD} 63$ was $2.91 \pm 0.19$ vs. $1.68 \pm 0.11, \mathrm{p}<0.05$. Similarly, $\Delta$ CD62P in ACS vs. SAP was $3.91 \pm 0.24$ vs. $2.43 \pm 0.18$, $\mathrm{p}<0.05$; and $\Delta$ CD63 was $2.91 \pm 0.19$ vs. $1.75 \pm 0.12, \mathrm{p}<0.05$. There was no significant difference between the controls and the SAP group. $\triangle \mathrm{CD} 62 \mathrm{P}$ in CTRL vs. SAP was $2.29 \pm 0.18$ vs. $2.43 \pm 0.18, \mathrm{p}=0.57$; and $\triangle \mathrm{CD} 63$ was $1.68 \pm 0.11$ vs. $1.75 \pm 0.12$, $\mathrm{p}=0.65$ (Fig. 7). The increases in CD62P and CD63 expression were positively correlated with the expression of TSLPR. For the correlation between TSLPR and the increase of the CD62P MFI: $r=0.41, p<0.01$; and for the correlation between TSLPR and the increase of the CD63 MFI: $r=0.34, p<0.01$ (Fig. 8, A-B).

\section{Discussion}

Platelet activation and acute thrombus formation in disrupted atherosclerotic plaques are important processes in the initiation of acute coronary syndromes [1-5]. Plaque rupture exposes thrombogenic components such as collagen, which could activate and link platelets, and the activated platelets may then release ADP to induce further platelet activation, ultimately leading to intravascular thrombus formation [1-3, 23-25]. Many studies have demonstrated that platelets contribute to immunoreactivity and can be activated by certain inflammatory factors [4- 6, 27]. In a preliminary study, we showed that TSLP promotes inflammation in atherosclerosis. TSLP is over-expressed in human atherosclerotic plaques, and the expression of TSLP can be induced by ox-LDL and angiotensin II $[8,9]$.

Our results revealed the presence of TSLPR on the surface of human platelets. TSLP was able to increase the expression of CD62P, CD63, and GPIIb/IIIa integrin, which could link to PAC-1 and induce platelet activation. CD62P, which is also known as P-Selectin, mediates the interaction of activated endothelial cells or platelets with leukocytes, which facilitates platelet-leukocyte aggregation and induces the formation of a platelet thrombus. CD63 is a member of the tetraspan transmembrane superfamily of proteins and is expressed on activated platelets. When platelets are activated, GPIIb/IIIa undergoes a conformational change to expose a ligand-binding site for adhesive macromolecules, including fibrinogen, von Willebrand factor, and fibronectin. Binding of fibrinogen to GPIIb/IIIa is required for platelet aggregation [23-25]. In this study, we demonstrated that TSLP alone was able to induce platelet aggregation and ATP release and markedly enhanced the aggregation and ATP release induced by collagen. In our investigation of the signalling channel mechanism, we found that the platelet activation induced by TSLP could be inhibited by an anti-TSLPR antibody and a PI3K inhibitor (LY294002). TSLP was able to enhance the phosphorylation of Akt. The phosphorylation level of AKT was significantly increased in platelets stimulated with TSLP and collagen together compared with platelets stimulated with collagen alone. This result showed that the mechanism underlying the TSLP-induced increase in collageninduced aggregation can be correlated with the enhancement of the PI3K/AKT pathway by TSLP. All of these results together suggested that TSLP can induce platelet activation through 
the TSLP/TSLPR signalling channel, via a mechanism that is most likely related to the PI3K/ AKT pathway.

To detect the potential link between the TSLP/TSLPR signalling channel with atherosclerosis and its complications, we tested the effects of TSLP on the platelets of CAD patients. We found that the expression of TSLPR, activation of platelets and sensitivity of TSLPR to TSLP were enhanced in the CAD patients (SAP and ACS groups) compared with the control group. In the SAP group, the expression of TSLPR and CD62P was markedly higher than in the controls. The expression and the increment of the expression of CD62P and CD63 induced by TSLP were positively correlated with the expression of TSLPR. All of these results suggested that the high expression level of TSLPR may be one of the reasons for the high activation status of platelets and high reactivity to TSLP observed in acute coronary syndrome. The TSLP/TSLPR signalling channel may be one of the mechanisms underlying platelet activation in atherothrombosis in coronary artery disease. In addition, these results indicate that patients showing higher TSLPR expression are more susceptible to developing ACS. Hence, in coronary artery disease patients, the determination of TSLPR in platelets may help to identify the risk of ACS, and in light of the comprehensive risk, necessary precautionary measures can be taken as early as possible.

The common clinically employed antiplatelet drugs, such as cyclooxygenase inhibitors (aspirin), ADP receptor antagonists (clopidogrel) and GPIIb/IIIa inhibitors (tirofiban), among others, are used either alone or in combination and are unable to completely prevent thrombopoiesis and ACS. In this context, our research results provide a new basis for targeting the TSLP/TSLPR signalling channel to limit platelet activation.

\section{Conflicts of Interest}

None declared.

\section{Acknowledgements}

This work was supported by a grant from the National Natural Science Foundation of China (No. 3114635) to Li Dazhu.

\section{References}

1 Jurk K, Kehrel BE: Pathophysiology and biochemistry of platelets. Intemist(Berl) 2010;51:1086-1094.

2 Leslie M: Cell biology. Beyond clotting: the powers of platelets. Science 2010;328:562-564.

3 Giovanni C, Paolo G, Vaccaro V, D'Anna M, Golino P: The missing link between atherosclerosis, inflammation and thrombosis: is it tissue factor. Expert Rev Cardiovasc Ther 2011;9:517-523.

-4 Semple JW, Freedman J: Platelets and innate immunity. Cell Mol Life Sci 2010;67:499-511.

5 Semple JW, Italiano JE Jr, Freedman J: platelets and the immune continuum. Nat Rev Immunol 2011;11:264274.

6 Hansson GK: Inflammation, atherosclerosis, and coronary arterydisease. N Engl J Med 2005;352:16851695.

7 Libby P, Ridker PM, Maseri A: Inflammation and Atherosclerosis. Circulation 2002;105:1135-1143.

-8 Lin J, Chang W, Dong J, Zhang F, Mohabeer N, Kushwaha KK, Wang L, Su Y, Fang H, Li D: Thymic Stromal Lymphopoietin Over-Expressed in Human Atherosclerosis: Potential Role in Th17 Differentiation. Cell Physiol Biochem 2013;31:305-318.

-9 Zhao H, Li M, Wang L, Su Y, Fang H, Lin J, Mohabeer N, Li D: Angiotensin II induces TSLP via an AT1 receptor/NF-KappaB pathway, promoting Th17 differentiation. Cell Physiol Biochem 2012;30:1383-1397. 


\section{Cellular Physiology $\quad$ Cell Physiol Biochem 2013;32:1741-1750}

and Biochemistry

Wang et al.: A Potential Role of Thymic Stromal Lymphopoietin in Platelet Activation in Acute Coronary Syndrome

10 Soumelis V, Reche PA, Kanzler H, Yuan W, Edward G, Homey B, Gilliet M, Ho S, Antonenko S, Lauerma A, Smith K, Gorman D, Zurawski S, Abrams J, Menon S, McClanahan T, de Waal-Malefyt Rd R, Bazan F, Kastelein RA, Liu YJ: Human epithelial cells trigger dendritic cell mediated allergic inflammation by producing TSLP. Nat Immunol 2002;3:673-680.

11 Sebastian K, Borowski A, Kuepper M, Friedrich K: Signal transduction around thymic stromal lymphopoietin (TSLP) in atopic asthma. Cell Commun Signal 2008;6:5.

12 Rochman Y, Leonard WJ: Thymic stromal lymphopoietin: a new cytokine in asthma. Curr Opin Pharmacol 2008;8:249-254.

13 Blagoev B, Nielsen MM, Angrist M, Chakravarti A, Pandey A: Cloning of rat thymic stromal lymphopoietinreceptor (TSLPR) and characterization of genomic structure of murine Tslpr gene. Gene 2002;284:161-168.

14 Pandey A, Ozaki K, Baumann H, Levin SD, Puel A, Farr AG, Ziegler SF, Leonard WJ, Lodish HF: Cloning of a receptor subunit required for signaling by thymic stromal lymphopoietin. Nat Immunol 2000;1:59-64.

15 Ying S, O'Connor B, Ratoff J, Meng Q Mallett K, Cousins D, Robinson D, Zhang G, Zhao J, Lee TH, Corrigan C: Thymic stromal lymphopoietin expression is increased in asthmatic airways and correlates with expression of Th2-attracting ahemokines and disease severity. J Immunol 2005;174:8183-8190.

16 Yoo J, Omori M, Gyarmati D, Zhou B, Aye T, Brewer A, Comeau MR, Campbell DJ, Ziegler SF: Spontaneous atopic dermatitis in mice expressing an inducible thymic stromal lymphopoietin transgene specifically in the skin. J Exp Med 2005;202:541-549.

17 Ziegler SF: Thymic stromal lymphopoietin and allergic disease. J Allergy Clin Immunol 2012;130:845-852.

18 Zhang W, Wang J, Wang Q, Chen G, Zhang J, Chen T, Wan T, Zhang Y, Cao X: Identification of a novel type I cytokine receptor CRL2 preferentially expressed by human dendritic cells and activated monocytes. Biochem Biophys Res Commun 2001;281:878-883.

19 Tonozuka Y, Fujio K, Sugiyama T, Nosaka T, Hirai M, Kitamura T: Molecular cloning of a human novel type I cytokine receptor related to delta1/TSLPR. Cytogenet Cell Genet 2001;93:23-25.

20 Reche PA, Soumelis V, Gorman DM, Clifford T, Liu Mr, Travis M, Zurawski SM, Johnston J, Liu YJ, Spits H, de Waal Malefyt R, Kastelein RA, Bazan JF: Human thymic stromal lymphopoietin preferentially stimulates myeloid cells. J Immunol 2001;167:336-343.

21 Ohba T, Haro H, Ando T, Koyama K, Hatsushika K, Suenaga F, Ohnuma Y, Nakamura Y, Katoh R, Ogawa H, Hamada Y, Nakao A: A potential role of thymic stromal lymphopoietin in the recruitment of macrophages to mouse intervertebral disc cells via monocyte chemotactic protein 1 induction: implications for herniated discs. Arthritis Rheum 2008;58:3510-3519.

22 Rochman Y, Leonard WJ: The role of thymic stromal lymphopoietin in CD8+ T cell homeostasis. J Immunol 2008;181:7699-7705.

23 Jackson SP. The growing complexity of platelet aggregation. Blood 2007;109:5087-5095.

24 Nurden A, Nurden P: Advances in our understanding of the molecular basis of disorders of platelet function. J Thromb Haemost 2011;1:76-91.

25 Kaushansky K: The molecular mechanisms that control thrombopoiesis. J Clin Invest 2005;115:3339-3347.

-26 Romaniuk MA, Tribulatti MV, Cattaneo V, Lapponi MJ, Molinas FC, Campetella O, Schattner M: Human platelets express and are activated by galectin-8. Biochem J 2010;432:535-547.

27 Zhang S, Yuan J, Yu M, Fan H, Guo ZQ, Yang R, Guo HP, Liao YH, Wang M: IL-17A facilitates platelet function through the ERK2 signaling pathway in patients with acute coronary syndrome. PLoS One 2012;7:e40641. 